\title{
Acquisition of STEM Images by Adaptive Compressive Sensing
}

\author{
Weiyi Xie ${ }^{1}$, Qianli Feng ${ }^{2}$, Ramprakash Srinivasan ${ }^{2}$, Andrew Stevens ${ }^{3,4}$, and Nigel D. Browning ${ }^{4,5}$
}

1. The Ohio State University, Dept. of Statistics, Columbus, OH, USA

2. The Ohio State University, Dept. of Electrical and Computer Engineering, Columbus, OH, USA

3. Duke University, Dept. of Electrical and Computer Engineering, Durham, NC, USA

4. Pacific Northwest National Laboratory, Richland, WA, USA

5. University of Washington, Dept. of Materials Science and Engineering, Seattle, Washington, USA

Compressive Sensing (CS) allows a signal to be sparsely measured first and accurately recovered later in software [1]. In scanning transmission electron microscopy (STEM), it is possible to compress an image spatially by reducing the number of measured pixels, which decreases electron dose and increases sensing speed $[2,3,4]$. The two requirements for CS to work are: (1) sparsity of basis coefficients and (2) incoherence of the sensing system and the representation system. However, when pixels are missing from the image, it is difficult to have an incoherent sensing matrix. Nevertheless, dictionary learning techniques such as Beta-Process Factor Analysis (BPFA) [5] are able to simultaneously discover a basis and the sparse coefficients in the case of missing pixels.

On top of CS, we would like to apply active learning [6,7] to further reduce the proportion of pixels being measured, while maintaining image reconstruction quality. Suppose we initially sample $10 \%$ of random pixels. We wish to select the next $1 \%$ of pixels that are most useful in recovering the image. Now, we have $11 \%$ of pixels, and we want to decide the next $1 \%$ of "most informative" pixels. Active learning methods are online and sequential in nature. Our goal is to adaptively discover the best sensing mask during acquisition using feedback about the structures in the image. In the end, we hope to recover a high quality reconstruction with a dose reduction relative to the non-adaptive (random) sensing scheme. In doing this, we try three metrics applied to the partial reconstructions for selecting the new set of pixels: (1) variance, (2) Kullback-Leibler (K-L) divergence using a Radial Basis Function (RBF) kernel, and (3) entropy.

Figs. 1 and 2 display the comparison of Peak Signal-to-Noise (PSNR) using these three different active learning methods at different percentages of sampled pixels. At $20 \%$ level, all the three active learning methods underperform the original CS without active learning. However, they all beat the original CS as more of the "most informative" pixels are sampled. One can also argue that CS equipped with active learning requires less sampled pixels to achieve the same value of PSNR than CS with pixels randomly sampled, since all the three PSNR curves with active learning grow at a faster pace than that without active learning when the pixel fraction is larger than $20 \%$. For this particular STEM image, by observing the reconstructed images and the sensing masks, we find that while the method based on RBF kernel acquires samples more uniformly, the one on entropy samples more areas of significant change, thus less uniformly. The K-L divergence method performs the best in terms of reconstruction error (PSNR) for this example [8]. 


\section{References:}

[1] E Candes and M Wakin. Signal Processing Magazine, IEEE. 2(25) (2008), pp. 21.

[2] A Stevens, H Yang, L Carin et al. Microscopy 63(1), (2014), pp. 41.

[3] L Kovarik, A Stevens, A Liyu et al. Appl. Phys. Lett. 109, 164102 (2016).

[4] A Stevens, L Kovarik, P Abellan et al. Adv. Structural and Chemical Imaging 1(10), (2015), pp. 1.

[5] M Zhou, H Chen, J Paisley et al. Image Processing, IEEE Transactions on 21(1), (2012), pp. 130.

[6] J Silva and L Carin. KDD 2012.

[7] S Ji, Y Xue, and L Carin. Signal Processing, IEEE Transactions on 56(6), pp. 2346.

[8] Supported by the Chemical Imaging, Signature Discovery, and Analytics in Motion Initiatives at PNNL. PNNL is operated by Battelle Memorial Inst. for the US DOE; contract DE-AC05-76RL01830.
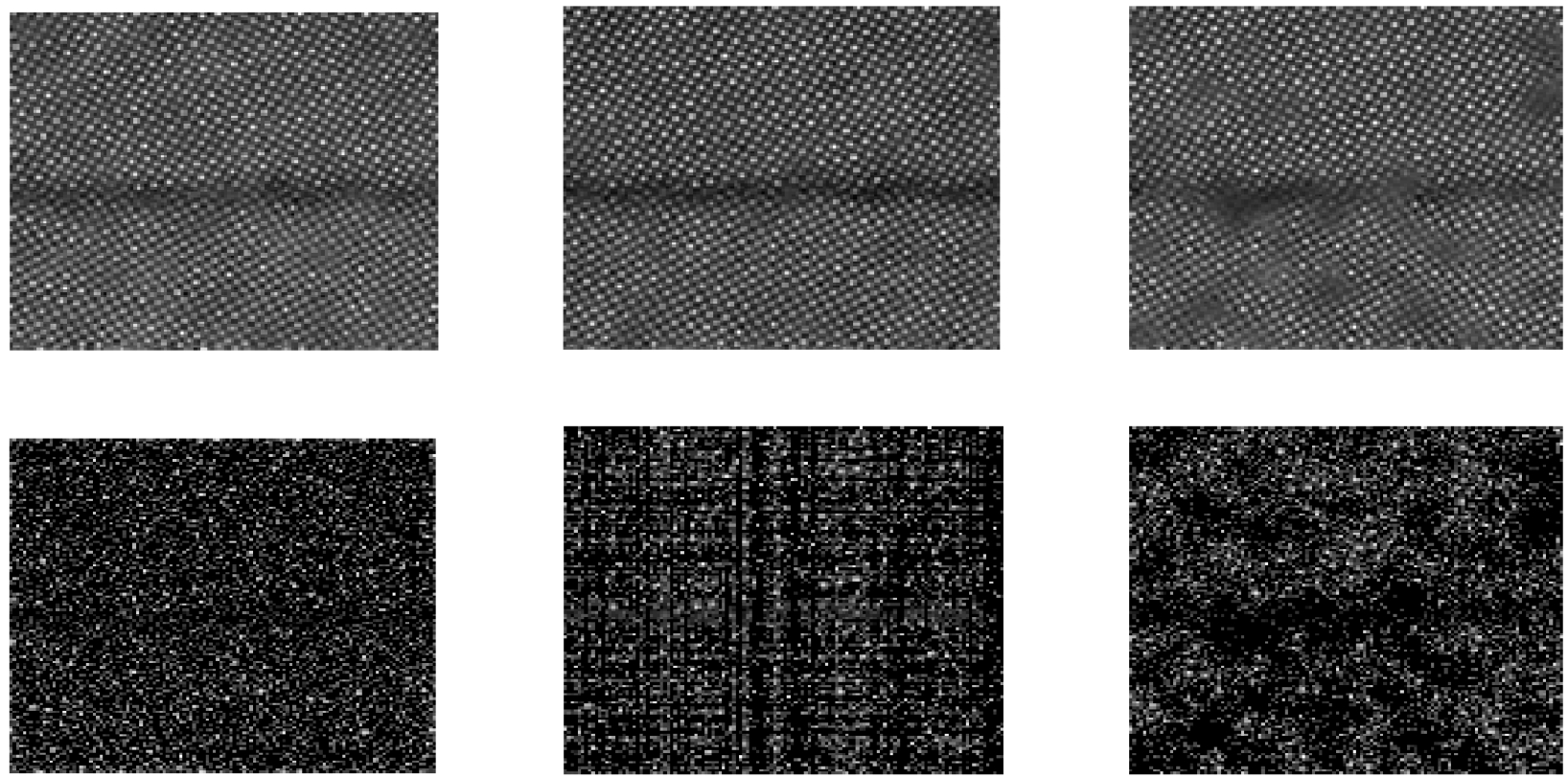

Figure 1. Reconstructed images (upper row) and sensing masks (lower row) by active learning based on variance (left column), RBF (middle column), and entropy (right column), up to $40 \%$ pixels sampled.

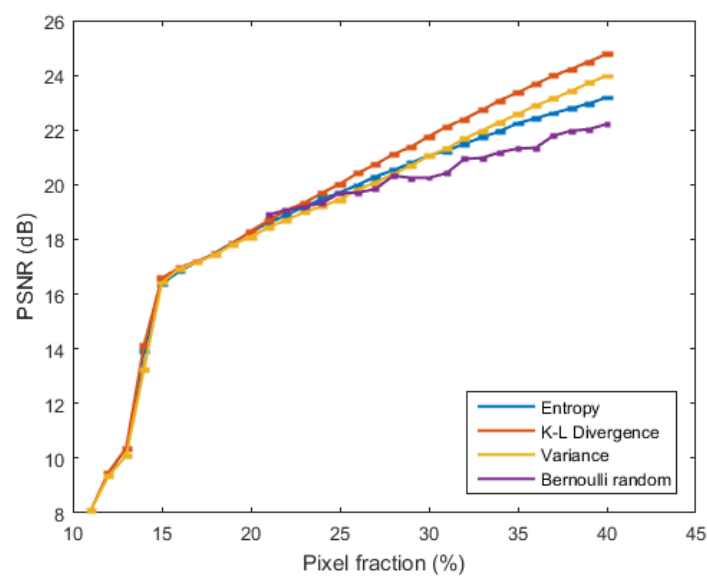

\begin{tabular}{|c|c|c|c|}
\hline Method \Pixel fraction & $20 \%$ & $30 \%$ & $40 \%$ \\
\hline Bernoulli random & 18.9 & 20.4 & 22.2 \\
\hline Variance & 18.4 & 21.3 & 24.0 \\
\hline K-L Divergence & 18.7 & 22.1 & 24.8 \\
\hline Entropy & 18.6 & 21.2 & 23.2 \\
\hline
\end{tabular}

Figure 2. Comparison of PSNR (dB) vs Pixel fraction (\%) for different methods. 